Review of

\title{
Interactive Multimedia Music Technologies
}

Edited by Kia $\mathrm{Ng}$ and Paolo Nesi

Information Science Reference

Hershey, PA

2008

$394 \mathrm{pp}$.

ISBN 978-159904150-6

US $\$ 180.00$ hard cover

Keywords Music - Data processing, Interactive multimedia, Music technology, Computers and music

Review DOI

Recent developments in interactive music technologies have profoundly changed and continue to influence the way music is rendered, published, distributed, taught and performed.

Author/editors Kia Ng (University of Leeds, UK) and Paolo Nesi (University of Florence, Italy) have assembled 14 chapters based on the research and findings of MUSICNETWORK, an international association that includes research institutions, music content providers, cultural heritage organizations and other music industry counterparts. Co-supported by the European Commission, the association strives to bring industry and related research areas together to focus on the development and application of state-of-the-art technologies designed to address the evolving and competitive landscape of the interactive multimedia music industry.

Following the editors brief introduction to the MUSICNETWORK's activities, services and working groups, the second and third chapters discuss the new emerging standard for modeling music notations within the MPEG multimedia framework called MPEG-SMR (Symbolic Music Representation). The next two chapters are dedicated to optical music imaging which investigates music digitization and recognition along with the architecture and algorithms both in use and under development.

Following chapters (6-7) are dedicated to address issues and offer proposals for designing XML based markup languages for music. It is argued that an XML application of music should enable nonprofessionals to distribute, create, play and 
organize music with the same simplicity that HTML enabled the average user with little computer knowledge to create web pages.

Chapters 8\&9 explore live music scenarios and the development of multimedia systems to integrate with live opera performance and experimentations in sound synthesis incorporating real-time audio signals.

The next pair of chapters focuses on technology and learning in both a cultural context as well as specific music tutoring systems. Chapters 12-13 discuss digital rights management technologies, the advantages and disadvantages that these technologies either provide or impose and what the future might hold for industry and consumers.

The final chapter concludes with an investigation and analyses of online music distribution, the major problems, different solutions and the various established business models.

This 394 page text includes a compilation of references, notes on the contributors and an index. The relatively short index is useful but could have benefited from a more comprehensive approach.

This excellent book will appeal to academic and research libraries, and those involved in the music technology and music networking industries. Music students, educators and audio visual artists interested in new and emerging multimedia music technologies will also benefit although much of the material is highly technical, requiring the reader to have advanced knowledge of computer science concepts. Some chapters, however, remain quite accessible to readers at a less technical level.

David Bindle 